
ohne ASS

Bei antikoagulierten Patienten, die einen Koronarstent benötigen, reicht Clopidogrel als Plättchenhemmer aus.

Die randomisierte WOEST-Studie, koordiniert von Prof. Willem Dewilde aus Tilburg, untersuchte zum ersten Mal bei 573 antikoagulierten Patienten, die aufgrund einer KHK einen Stent erhielten, ob bei diesen Patienten auf ASS verzichtet werden kann. Die Hälfte der Patienten erhielt einen Vitamin-K-Antagonisten plus Clopidogrel, die andere Hälfte zusätzlich ASS. Das Follow-up betrug ein Jahr. Die Patienten, bei denen ASS weggelassen wurde, hatten nicht nur ein geringeres Blutungsrisiko (19,5 vs 44,9\%), sondern auch einen signifikanten Mortalitätsvorteil (2,6 versus $6,4 \%$ Verstorbene nach einem Jahr). Außerdem waren Herzinfarkte (3,3 versus 4,7\%), Schlaganfälle (1,1 vs $2,9 \%)$ und StentThrombosen (1,5 vs 3,2\%) unter dem Zwei-Medikamenten-Regime seltener als unter der Triple-Therapie.

\title{
Schützt Selen vor Prostatakrebs?
}

\author{
Können sich Männer mit der Einnahme von \\ Selenpräparaten vor Prostatakrebs schüt- \\ zen? Der Effekt hängt offenbar von der \\ richtigen Dosis ab.
}

Wie eine aktuelle Metaanalyse nahelegt, ist die schützende Wirkung von Selen wahrscheinlich eine Frage der richtigen Dosis. Das Team um Dr. Rachel Hurst von der University of East Anglia in Norwich wertete zwölf Studien mit insgesamt über 13.000 Teilnehmern aus und analysierte das Prostatakarzinomrisiko in Abhängigkeit von der Selen-Plasma-Konzentration. Ergebnis: Innerhalb des relativ engen Bereichs zwischen 60 und $170 \mathrm{ng} / \mathrm{ml}$ nahm das Erkrankungsrisiko mit steigenden Selenwerten um bis zu 25\% ab. Das relative Risiko betrug beispielsweise bei $135 \mathrm{ng} / \mathrm{ml} \mathrm{0,85} \mathrm{und} \mathrm{bei} 170 \mathrm{ng} / \mathrm{ml} \mathrm{0,75.} \mathrm{(170} \mathrm{ng} / \mathrm{ml}$ war der höchste Wert, der in den Studien gemessen wurde). Die Frage wie viel Selen „Mann“ zu sich nehmen muss, um sein Prostatakrebsrisiko zu senken, bleibt dennoch ungeklärt. 